\title{
UTILIZAÇÃO DA ESTATÍSTICA MULTIVARIADA COMO FERRAMENTA PARA IDENTIFICAÇÃO DAS POSSÍVEIS FONTES DE POLUIÇÃO DO ARROIO LAVRAS DO SUL/RS, BRASIL
}

\author{
Luana Nunes Centeno ${ }^{1}$, Samanta Tolentino Cecconello ${ }^{2}$, Hugo Alexandre Soares Guedes ${ }^{3}$, Diuliana Leandro ${ }^{4}$ \\ 1 Mestranda em Recursos Hídricos, Universidade Federal de Pelotas, 96010-610, Pelotas, Brasil. \\ 2 Mestranda em Desenvolvimento Territorial e Sistemas Agroindustriais, Universidade Federal de Pelotas, 96010-610, Pelotas, Brasil. \\ 3 Docente do PPG em Recursos Hídricos, Universidade Federal de Pelotas, 96010-610, Pelotas, Brasil. \\ ${ }^{4}$ Docente do Centro de Engenharias, Universidade Federal de Pelotas, 96010-610, Pelotas, Brasil. \\ *E-mail: luananunescenteno@gmail.com
}

\section{RESUMO}

A poluição existente em um corpo d'água é fortemente influenciada pelas diversas atividades antrópicas existentes na bacia hidrográfica da qual o manancial faz parte, demandando a identificação das fontes de poluição para que esses corpos hídricos sejam adequados aos usos a que se dispõem. Diante disso, este trabalho objetivou utilizar a análise fatorial/componentes principais para identificar as possíveis fontes de poluição do arroio Lavras do Sul, localizado no sul do Estado do Rio Grande do Sul. Para isso, foram utilizadas nove variáveis de qualidade da água disponibilizadas pela Fundação Estadual de Proteção Ambiental Henrique Luis Roessler, sendo elas: Oxigênio Dissolvido (OD), Demanda Bioquímica de Oxigênio $\left(\mathrm{DBO}_{5}{ }^{20}\right)$, Turbidez (TH), Nitrogênio Total Kjeldahl (NTK), Fósforo Total (PT), Temperatura da Água $\left(\mathrm{TH}_{2} \mathrm{O}\right)$, Coliformes Termotolerantes (CT), Sólidos Totais (ST) e Potencial Hidrogeniônico $(\mathrm{pH})$. As variáveis que apresentaram os maiores coeficientes de correlação com outras variáveis envolvidas neste estudo foram $\mathrm{CT}, \mathrm{DBO}_{5}^{20}$, OD, $\mathrm{TH}_{2} \mathrm{O}$ e TH. Após a decomposição da matriz de correlação de variáveis obtiveram-se dois fatores que explicaram $100 \%$ da variância total dos dados. A primeira componente principal foi relacionada com atividades provenientes de ações antrópicas, como despejos de esgotos domésticos e atividades agropecuárias. Já a segunda componente principal foi relacionada a despejos de efluentes industriais e atividades de mineração.

Palavras-chave: Análise de Componentes Principais. Arroio Lavras do Sul/RS. Qualidade da Água

\section{Introdução}

Ao longo dos anos, observa-se com maior frequência, principalmente em estudos relacionados aos recursos hídricos, a preocupação não só com a quantidade de água, mas também com a qualidade dos mananciais. Uma das causas são as atividades antrópicas, que vêm contribuindo acentuadamente para a entrada de compostos, seja de forma difusa, seja pontual, nos corpos hídricos [1-3].

A urbanização e as atividades industriais caracterizamse por serem importantes agentes poluidores pontuais nas bacias hidrográficas. $\mathrm{O}$ esgoto doméstico, rico em matéria orgânica, é lançado in natura nos corpos d'água e, dependendo da relação entre as vazões de esgoto lançado e a do corpo receptor, pode resultar em sérios prejuízos à qualidade da água, como toxicidade e proliferação de algas [4]. As práticas agrícolas destacam-se como os principais agentes poluidores difusos, que contribuem com o aumento de nutrientes nos corpos hídricos, principalmente fósforo e nitrogênio, favorecendo os processos de eutrofização [5].

Com a degradação dos recursos hídricos há a necessidade de se avaliar, e principalmente monitorar, a qualidade da água, a fim de fornecer uma indicação das alterações induzidas pelas atividades antrópicas; compreender os processos naturais de uma bacia hidrográfica e avaliar a adequação da água para os usos pretendidos [4,6].

O monitoramento ambiental serve como medida de apoio para tomadas de decisão, pois é possível acompanhar a evolução das variáveis limnológicas de qualidade da água ao longo do tempo e do espaço [7,8]. Porém, o monitoramento 
ambiental de um manancial, por envolver diversas variáveis de qualidade da água, demanda tempo e altos investimentos financeiros, o que muitas vezes inviabiliza o processo. Além do mais, não é possível se obter uma visão integral do que realmente está ocorrendo em um corpo hídrico ao se analisar cada variável limnológica isoladamente $[9,10]$.

Uma forma de analisar simultaneamente várias variáveis de qualidade da água, ao longo do tempo e do espaço, é através da estatística multivariada. Essa técnica estatística vem sendo empregada em diversos estudos ambientais [11-15]. Dentre os diversos métodos estatísticos multivariados passíveis de serem utilizados, há a Análise de Componentes Principais (ACP) e a Análise Fatorial (AF) [17, 18]. Esses métodos foram introduzidos por Karl Pearson, em 1901, com o objetivo principal de reduzir o número de variáveis originais em um conjunto menor de variáveis (denominados de fatores), não correlacionadas entre si, com uma perda mínima de informações [18]. Esses fatores gerados, normalmente, são empregados para explicar o relacionamento entre um grupo de variáveis [16].

Zimmermann, Guimarães e Peralta-Zamora [27] avaliaram a qualidade da água do rio Tibagi, na região de Ponta Grosa/PR, utilizando a ACP. Os autores encontraram como principal fonte de poluição as atividades industriais. Guedes et al. [28], aplicando a técnica de ACP no curso médio do Rio Pomba/MG, obtiveram três componentes principais que explicaram 74,3\% da variância total dos dados, estando associadas a despejos domésticos, a sólidos em suspensão e a processos erosivos. Outros autores [29,30] encontraram resultados semelhantes a esse estudo quando utilizaram as mesmas técnicas de análise estatística multivariada para avaliarem a qualidade da água.

Diante disso, este trabalho objetivou utilizar a análise fatorial/analise de componentes principais para identificar as possíveis fontes de poluição do arroio Lavras do Sul, localizado no sul do Estado do Rio Grande do Sul.

\section{Materiais e Métodos}

\subsection{Caracterização da Área de Estudo}

A Bacia Hidrográfica do Litoral é formada pelas bacias do leste e do extremo sul do Estado e é dividida em cinco bacias, sendo elas: Tramandaí, Litoral Médio, Camaquã, Piratini-São Gonçalo e Mampituba. A Bacia Hidrográfica Camaquã (BHC), situada na porção centro-leste do Estado do Rio Grande do Sul, a $28^{\circ} 50^{\prime}$ e $30^{\circ} 00^{\prime}$ Sul e $52^{\circ} 15^{\prime}$ e $53^{\circ} 00^{\prime}$ Oeste, abrange o município de Lavras do Sul - RS (Fig. 1).

Figura 1: Localização geográfica da área de estudo.

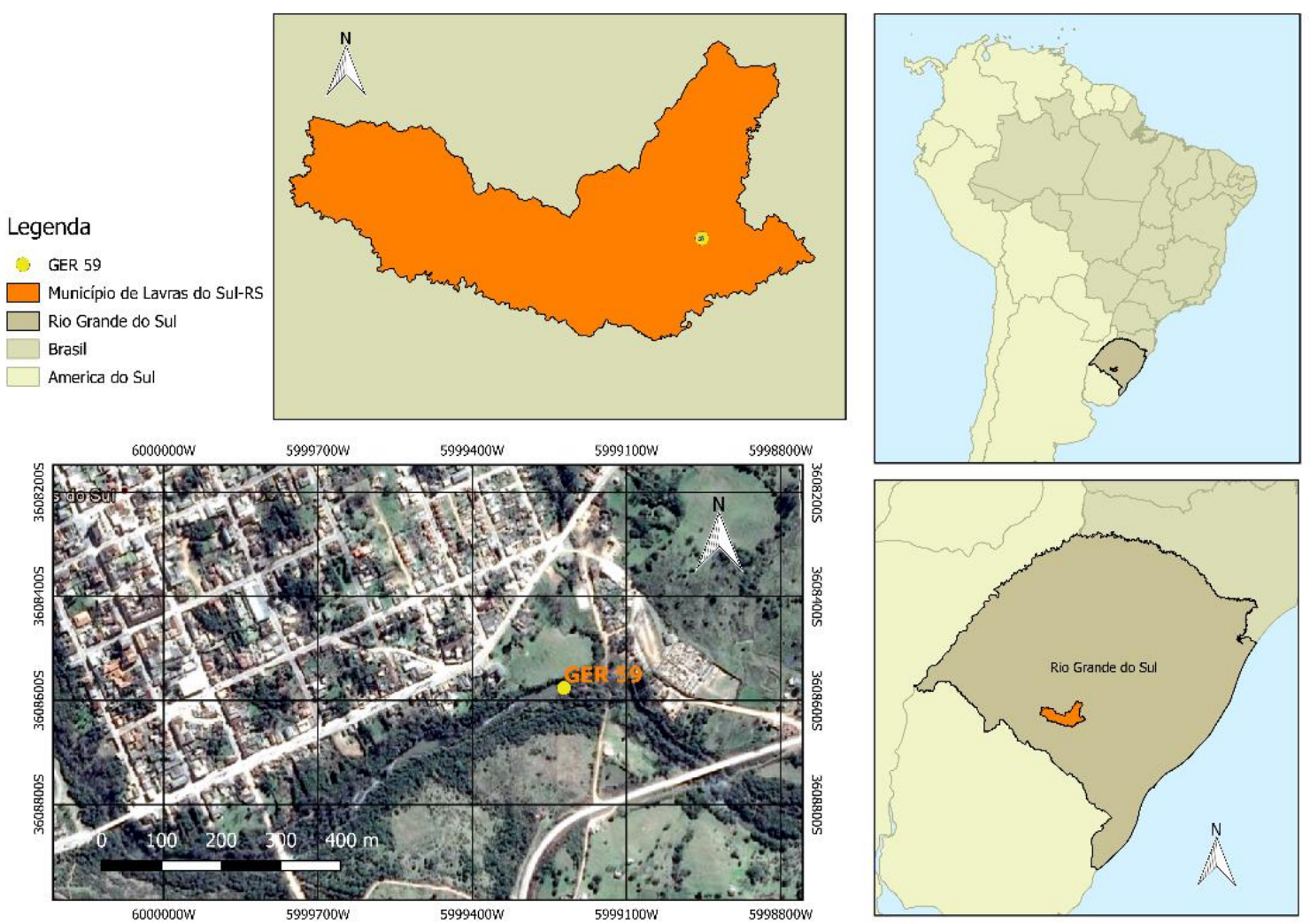


Segundo o Comitê de Gerenciamento da Bacia Hidrográfica do Rio Camaquã (CGBHRC), as nascentes da bacia estão situadas próximo às localidades de Torquato Severo, no município de Dom Pedrito, divisa com o município de Bagé, e Tabuleiro, no município de Lavras do Sul. O ponto de monitoramento, objeto deste estudo, é o GER 59, localizado no arroio Lavras do Sul (Fig. 1), no Alto Camaquã, no município de Lavras do Sul, situado geograficamente na Latitude 30 50'09” Sul e Longitude 5348'0.56" Oeste [19], sendo que no município há atividades mineradoras [20].

\subsection{Base de Dados}

A Fundação de Proteção Ambiental Henrique Luis Roessler (FEPAM/RS) é responsável pelo monitoramento da qualidade dos corpos d'água da Região da Bacia do Litoral desde 1992. As coletas e análises de água ocorrem a cada seis meses, contemplando os períodos chuvoso e seco. Foram utilizados neste estudo os dados de qualidade da água disponibilizados pela FEPAM/RS compreendidos entre os anos de 2005 a 2013.

As variáveis de qualidade de água utilizadas, por apresentarem baixa percentagem de falhas amostrais, foram: Demanda Bioquímica de Oxigênio $\left(\mathrm{DBO}_{5}{ }^{20}\right)$, Coliformes Termotolerantes (CT), Fósforo Total (PT), Nitrogênio Total Kjeldahl (NTK), Oxigênio Dissolvido (OD), Turbidez (TH), Potencial Hidrogeniônico $(\mathrm{pH})$, Temperatura da água $\left(\mathrm{TH}_{2} \mathrm{O}\right)$ e Sólidos Totais (ST).

As coletas foram realizadas pela FEPAM seguindo a metodologia descrita pela Companhia Ambiental do Estado de São Paulo [21]. Os métodos analíticos, bem como a preservação das amostras, seguiram os procedimentos definidos por APHA [22].

\subsection{Análise Estatística}

A ligação entre as variáveis que influenciam a qualidade da água do arroio Lavras do Sul foi identificada através da Análise de Componentes Principais (ACP) e da Análise Fatorial (AF). Para a realização da normalização dos dados, bem como das análises estatísticas, utilizou-se o software Statistica ${ }^{\circledR}$, versão 7.0.

Foi necessário realizar a normalização dos dados, pois as variáveis apresentavam unidade de medidas distintas [23,32]. Essa normalização foi feita pelo próprio software, através do desvio-padrão de cada variável. Por conseguinte, construiu-se a matriz de dados definida por $\mathrm{X}=\left(\mathrm{x}_{\mathrm{i}, \mathrm{j}}\right)$, em que "i” é igual ao número de variáveis, ou seja, "9" e "j” é o número de elementos amostrais, ou seja, "16". Sendo assim, a matriz original foi de 9X16, que foi transformada em seguida em uma matriz de correlações $[R]$ (pxp), em que "p" se define como as 9 variáveis de qualidade da água do arroio Lavras do Sul.

Após a geração da matriz de correlação, seguiu-se a sua decomposição com o objetivo de identificar as possíveis fontes de poluição existentes no arroio Lavras do Sul. Isso foi possível através da geração das componentes principais (CPs), a partir do número de fatores que representariam o que estava ocorrendo no manancial.

Para a definição do número dos fatores utilizou-se a metodologia descrita por Corrar et al. [16] e Jolliffe [24] que diz que a máxima variabilidade total dos dados deve ser explicada através das três primeiras componentes, seguindo a seguinte regra: a primeira componente principal não pode ter correlação com a segunda e a segunda não pode estar correlacionada com a terceira, e esta não pode estar correlacionada nem com a primeira nem com a segunda, e assim sucessivamente, até que as CPs expliquem mais que 70\% da variância total dos dados [23,31].

\section{Resultados e discussões}

Na Tabela 1 está apresentada a matriz de correlação das variáveis de qualidade da água do arroio Lavras do Sul. Observa-se na Tabela 1 que as variáveis $\mathrm{CT}, \mathrm{DBO}_{5}{ }^{20}$, OD, $\mathrm{TH}_{2} \mathrm{O}$ e TH apresentaram maiores coeficientes de correlação com maior número de variáveis.

Constatou-se correlação muito forte e positiva entre as variáveis TH e CT $(\mathrm{r}=0,998), \mathrm{PT}$ e $\mathrm{DBO}_{5}{ }^{20}(\mathrm{r}=0,998), \mathrm{pH}$ e $\mathrm{TH}_{2} \mathrm{O}(\mathrm{r}=0,995)$, NTK e OD $(\mathrm{r}=0,852)$, OD e CT $(\mathrm{r}=0,809)$, OD e TH $(r=0,774)$, TH e $\mathrm{DBO}_{5}{ }^{20}(\mathrm{r}=0,756), \mathrm{DBO}_{5}{ }^{20}$ e CT $(r=0,717)$ e PT e TH $(r=0,712)$.

A correlação muito forte positiva ente o $\mathrm{pH}$ e $\mathrm{TH}_{2} \mathrm{O}$ se justifica, pois quando ocorre o aumento da temperatura elevamse as taxas de reações químicas, o que torna possível a elevação do $\mathrm{pH}$.

Já os CT têm alta correlação positiva com $\mathrm{OD}, \mathrm{DBO}_{5}{ }^{20}$ e $\mathrm{TH}$, pois conforme aumenta a presença desta variável, aumenta a quantidade de matéria orgânica no manancial, o que por sua vez tem relação direta com a $\mathrm{DBO}_{5}{ }^{20}$ e o OD. Os CT associados à matéria orgânica alteram a cor aparente da água, deixando-a mais turva [3].

Os nutrientes contidos na água, como PT e NTK, também têm relação direta com a matéria orgânica, pois o PT e o NTK são nutrientes indispensáveis para o crescimento dos micro-organismos, que por sua vez são responsáveis pela estabilização da matéria orgânica. Porém, esses nutrientes, quando em elevadas concentrações, podem causar a eutrofização dos corpos hídricos e aumentar a turbidez da água $[2,10]$.

As correlações foram muito fortes e negativas entre as variáveis: $\mathrm{TH}_{2} \mathrm{O}$ e $\mathrm{OD}(\mathrm{r}=-0,985)$, $\mathrm{pH}$ e $\mathrm{OD}(\mathrm{r}=-0,963)$, NTK e ST $(r=-0,961)$, CT e pH $(r=-0,936)$, pH e TH $(r=-0,914)$, 
CT e $\mathrm{TH}_{2} \mathrm{O}(\mathrm{r}=-0,897), \mathrm{TH}_{2} \mathrm{O}$ e $\mathrm{TH}(\mathrm{r}=-0,871)$ e $\mathrm{TH}_{2} \mathrm{O}$ e

NTK $(r=-0,751)$.

\begin{tabular}{|c|c|c|c|c|c|c|c|c|c|}
\hline & CT & $\mathrm{DBO}_{5}{ }^{20}$ & PT & NTK & OD & $\mathrm{pH}$ & ST & $\mathrm{TH}_{2} \mathrm{O}$ & TH \\
\hline CT & 1,000 & & & & & & & & \\
\hline $\mathrm{DBO}_{5}{ }^{20}$ & 0,717 & 1,000 & & & & & & & \\
\hline PT & 0,670 & 0,998 & 1,000 & & & & & & \\
\hline NTK & 0,383 & $-0,369$ & $-0,428$ & 1,000 & & & & & \\
\hline OD & $\mathbf{0 , 8 0 9}$ & 0,171 & 0,107 & $\mathbf{0 , 8 5 2}$ & 1,000 & & & & \\
\hline $\mathrm{pH}$ & $-0,936$ & $-0,427$ & $-0,368$ & $-0,682$ & $-0,963$ & 1,000 & & & \\
\hline ST & $-0,115$ & 0,609 & 0,659 & $-0,961$ & $-0,677$ & 0,455 & 1,000 & & \\
\hline $\mathrm{TH}_{2} \mathrm{O}$ & $-0,897$ & $-0,336$ & $-0,274$ & $-0,751$ & $-0,985$ & 0,995 & 0,541 & 1,000 & \\
\hline $\mathrm{TH}$ & 0,998 & 0,756 & 0,712 & 0,329 & 0,774 & $-0,914$ & $-0,057$ & $-0,871$ & 1,000 \\
\hline
\end{tabular}

$\mathrm{DBO}_{5}{ }^{20}$ : Demanda Bioquímica de Oxigênio, CT: Coliformes Termotolerantes, FT: Fósforo Total, NTK: Nitrogênio Total Kjeldahl, OD: Oxigênio Dissolvido, TH: Turbidez, pH: Potencial Hidrogeniônico, $\mathrm{TH}_{2} \mathrm{O}$ : Temperatura da água e ST: Sólidos Totais.

A correlação entre as variáveis ST e NTK era esperada, uma vez que o NTK pode ser advindo das dissoluções de sais minerais. Segundo Von Sperling [3] esse nutriente contribui para o aumento das concentrações de sólidos totais. A correlação muito forte negativa do $\mathrm{pH}$ com as demais variáveis pode ser decorrente das interferências antrópicas, o mesmo ocorrendo com a autocorrelação existente entre as variáveis correlacionadas com $\mathrm{TH}_{2} \mathrm{O}$ [2].

Bertossi et al. [25] necessitaram de três fatores para explicar $87,5 \%$ da variabilidade da qualidade da água de uma sub-bacia hidrográfica rural do Estado do Espirito Santo. No presente estudo, entretanto, necessitou-se de apenas dois fatores para explicar 100\% da variância total dos dados (Tabela 2).

Tabela 2 - Fatores relacionados à variância amostral

\begin{tabular}{ccccc}
\hline Fator & Autovalor & $\begin{array}{c}\text { Variância } \\
\text { Total } \\
\text { Explicada }(\%)\end{array}$ & $\begin{array}{c}\text { Autovalor } \\
\text { Acumulado }\end{array}$ & $\begin{array}{c}\text { Variância } \\
\text { Total } \\
\text { Acumulada (\%) }\end{array}$ \\
\hline 1 & 5,637326 & 62,63695 & 5,637326 & 62,6370 \\
2 & 3,362674 & 37,36305 & 9,000000 & 100,0000 \\
\hline
\end{tabular}

Na Tabela 3 está apresentada a matriz de peso fatorial das variáveis de qualidade de água associada às duas componentes principais selecionadas.

A componente principal $1\left(\mathrm{CP}_{1}\right)$, responsável por $62,6 \%$ da variância total dos dados (Tab. 2), foi representada pelas variáveis $\mathrm{CT}, \mathrm{DBO}_{5}{ }^{20}, \mathrm{OD}, \mathrm{pH}, \mathrm{TH}_{2} \mathrm{O}$ e $\mathrm{TH}$ (Tab. 3). Essa componente indica interferência de origem antropogênica na poluição do arroio, como despejos domésticos, atividades agropecuárias ou manejo e adubação orgânica inadequada, erosão hídrica, ressuspensão de sedimentos e descartes inadequados de resíduos.

Já a componente principal $2\left(\mathrm{CP}_{2}\right)$, responsável por 37,4\% da variância total dos dados (Tab. 2), é explicada através das variáveis PT, NTK e ST (Tab. 3). A relação entre essas três variáveis traz como possíveis fontes de poluição os despejos industriais, as atividades agrícolas, os detergentes e as atividades mineradoras. Com relação a possíveis fontes de poluição natural, a $\mathrm{CP}_{2}$ pode ter relação com a dissolução de compostos do solo, a decomposição da matéria orgânica, o fósforo e o nitrogênio - em função da decomposição celular de micro-organismos - dentre outros constituintes de proteínas e compostos biológicos [2,26].

Tabela 3 - Matriz de peso fatorial das variáveis da qualidade de água

\begin{tabular}{ccc} 
Variáveis & $\mathrm{CP}_{1}$ & $\mathrm{CP}_{2}$ \\
\hline $\mathrm{CT}$ & $\mathbf{0 , 9 5 9 7 0 2}$ & $-0,281019$ \\
$\mathrm{DBO}_{5}{ }^{20}$ & $\mathbf{- 0 , 8 7 0 3 2 9}$ & 0,492471 \\
$\mathrm{PT}$ & 0,435354 & $\mathbf{- 0 , 9 0 0 2 5 9}$ \\
$\mathrm{NTK}$ & 0,627248 & $\mathbf{0 , 7 7 8 8 2 0}$ \\
$\mathrm{OD}$ & $\mathbf{0 , 9 4 1 6 0 8}$ & 0,336712 \\
pH & $\mathbf{- 0 , 9 9 7 2 9 1}$ & $-0,073554$ \\
$\mathrm{ST}$ & $-0,389249$ & $\mathbf{- 0 , 9 2 1 1 3 2}$ \\
$\mathrm{TH}{ }_{2} \mathrm{O}$ & $\mathbf{- 0 , 9 8 5 2 0 7}$ & $-0,171366$ \\
$\mathrm{TH}$ & $\mathbf{0 , 9 4 2 0 3 7}$ & $-0,335509$
\end{tabular}

$\mathrm{DBO}_{5}{ }^{20}:$ Demanda Bioquímica de Oxigênio, CT: Coliforme Termotolerantes, FT: Fósforo Total, NTK: Nitrogênio Total Kjeldahl, OD: Oxigênio Dissolvido, TH: Turbidez, pH: Potencial Hidroiônico, $\mathrm{TH}_{2} \mathrm{O}$ : Temperatura da Água e ST: Sólidos Totais.

\section{Conclusões}

Através deste estudo foi possível concluir que duas componentes principais foram suficientes para explicar $100 \%$ da variância total dos dados de qualidade da água. A primeira componente principal foi relacionada com atividades provenientes de ações antrópicas, como despejos de esgotos domésticos e atividades agropecuárias. Já a segunda componente principal foi relacionada a despejos de efluentes industriais e atividades de mineração. 


\section{Agradecimentos}

Os autores agradecem à CAPES (Coordenação de Aperfeiçoamento de Pessoal de Nível Superior), pela concessão da bolsa de estudo durante todo o período de realização do curso de mestrado do primeiro autor, que está sendo realizado no PPG em Recursos Hídricos/UFPEL, e também à Fepam (Fundação Estadual de Proteção Ambiental Henrique Roessler), por disponibilizar os dados para o desenvolvimento desta pesquisa.

\section{USE OF MULTIVARIATE STATISTICS AS A TOOL FOR IDENTIFYING POSSIBLE SOURCES OF POLLUTION OF THE LAVRAS DO SUL RIVER/RS, BRAZIL}

ABSTRACT: The pollution present in a stream is strongly influenced by several human activities existing in the watershed which the stream is a part of, requiring the identification of sources of pollution, so that the stream can keep suitable for use. Thus, this study aimed to use the factor analysis/principal components to identify possible sources of pollution of the Lavras do Sul river, located in the southern part of the Rio Grande do Sul State, Brazil. In order to do that, we used nine water quality variables provided by the Fundação Estadual de Proteção Ambiental Henrique Luis Roessler. These are: Dissolved Oxygen (DO), Biochemical Oxygen Demand $\left(\mathrm{BOD}_{5}{ }^{20}\right)$, Turbidity (TH), Total Kjeldahl Nitrogen (TKN), Total Phosphorus (TP), Water Temperature $\left(\mathrm{H}_{2} \mathrm{OT}\right)$, Thermotolerant Coliforms (TC), Total Solids (TS) and Hydrogen Potential (pH). The variables that presented the highest correlation coefficients with other variables involved in this study were $\mathrm{TC}, \mathrm{BOD}_{5}{ }^{20}, \mathrm{DO}$, $\mathrm{H}_{2} \mathrm{OT}$ and $\mathrm{TH}$. After decomposing the variable correlation matrix two factors that explained $100 \%$ of the total variance of data were obtained. The first principal component was related to human actions, as the throwing of domestic sewage and agricultural activities. The second principal component was related to the throwing of industrial waste and mining activities.

Keywords: Principal Component Analysis. Lavras do Sul river/RS. Water Quality.

\section{Referências}

[1] ABREU, C. H. M.; CUNHA, A. C. Qualidade da Água em Ecossistemas Aquáticos Tropicais Sob Impactos Ambientais no Baixo Rio Jari-AP: Revisão Descritiva. Biota Amazônia, v. 5, n. 2, p. 119-131, 2015. Disponível em: <http://periodicos.unifap.br/index.php/biota/article/view/1294/v5n2p119131.pdf $>$. Acesso em: 13 jun. 2016.

[2] BRAGA, B.; TUNDISI, J.G; TUNDISI, T. M.; CIMINELLI, V. S. T. Águas Doces no Brasil: capital ecológico, uso e conservação. 4. ed. São Paulo: Escrituras, 2015.
[3] VON SPERLING, M. Introdução à Qualidade das Águas e ao Tratamento de esgotos. 3. ed. Belo Horizonte: DESA - UFMG, v. 1, 2005. 452 p.

[4] NUVOLARI, A. Esgoto sanitário: coleta, transporte, tratamento e reuso agrícola. São Paulo, Edgard Blucher, 520p. 2003.

[5] SARDINHA, D. S.; CONCEIÇÃO, F. T.; SOUZA, A. D.; SILVEIRA, A.; JULIO, M.; GONÇALVES, J. C. S.I. Avaliação da qualidade da água e autodepuração do ribeirão do meio, Leme (SP). Engenharia Sanitária e Ambiental [online], v.13. n. 3, p. 329-338, jul./set. 2008. Disponível em: $<$ http://www.scielo.br/scielo.php?pid=S1413-

41522008000300013\&script=sci_arttext $>$. Acesso em: 15 jun. 2016.

[6] HELLER, L.; PÁDUA, L. Abastecimento de água para consumo humano. 2. ed. Belo Horizonte: UFMG, v. 1, 2010.

[7] TAVARES, B. S. Qualidade de água na bacia hidrográfica do rio Una Pernambuco. 2014. 110 f. Tese (Doutorado em Engenharia Agrícola) Universidade Federal Rural de Pernambuco. Recife, 2014. Disponível em: <http://www.condepefidem.pe.gov.br/c/document_library/get_file?p_1_id=78673 $\&$ folderId=141847 \&name=DLFE-11996.pdf $>$. Acesso em: 23 jul. 2016.

[8] TOMAZONI, Julio Caetano; RODRIGUES, Marilucia Cyrino; GUIMARÃES, Elisete. Qualidade da Água da Bacia do Rio Capivara, Manancial de Abastecimento do Município de São João - PR. Revista Brasileira de Geografia Física, São João, v. 8, n. 1, p.81-92, 24 fev. 2015. Disponível em: 〈http://www.revista.ufpe.br/rbgfe/index.php/revista/article/viewArticle/1123〉. Acesso em: 25 jul. 2016.

[9] GOMES, A. I; PIRES, J.C.M; FIGUEIREDO, S. A; BOAVENTURA, R. A.R. Optimization of River Water Quality Surveys by Multivariate Analysis of Physicochemical, Bacteriological and Ecotoxicological Data. Water Resources Management, v. 28, n. 5, p. 1345-1361, 2014. Disponível em: <http://link.springer.com/article/10.1007/s11269-014-0547-9>. Acesso em: 16 jul. 2016.

[10] LIBÂNIO, M. Fundamentos de Qualidade e Tratamento de Água. Campinas: Átomo, 2010.

[11] AL-MUTAIRI, N.; ABAHUSSAIN, A.; EL-BATTAY, A. Spatial and temporal characterizations of water quality in Kuwait Bay. Marine Pollution Bulletin, v. 83, n. 1, p. 127-131, 2014. Elsevier Ltd. Disponível em: <http://www.sciencedirect.com/science/article/pii/S0025326X14002124>.

Acesso em: 13 jul. 2016.

[12] GOMÉZ, D. M.; Estudio del modelamiento de la calidad del agua del río Sinú, Colombia. Revista de Ingenierías: Universidad de Medellín, v. 12, n. 22, p. 33-44, jan./jun.2013. DisponÍvel em:< http://www.scielo.org.co/scielo.php?pid=S1692-

33242013000100004\&script=sci_arttext> Acesso em 07 jul. 2016.

[13] IANG, K. et al. Multivariate statistical methods and Six-Sigma. Journal. Six Sigma and Competitive Advantage, v.1, n. 1, p. 76-96, 2004. Disponível em: http://www.researchgate.net/publication/239433549_Multivariate_statistical_met hods_and_Six-Sigma. Acesso em: 22 jul. 2016.

[14] MUSTONEN, S. M; TISSARI, S; HUIKKO, L; KOLEHMAINEN, M; LEHTOLA, M.J; HIRVONEN, A. Evaluating online data of water quality changes in a pilot drinking water distribution system with multivariate data exploration methods. Water Research, v. 42, n. 10-11, p. 2421-2430, 2008 Disponível

<http://linkinghub.elsevier.com/retrieve/pii/S0043135408000328>. Acesso em: 03 jun. 2016.

[15] OLSEN, R. L.; CHAPPELL, R. W.; LOFTIS, J. C. Water quality sample collection, data treatment and results presentation for principal components analysis - literature review and Illinois River watershed case study. Water Research, v. 46, n. 9, p. 3110-3122, 2012. Disponível em: <http://dx.doi.org/10.1016/j.watres.2012.03.028>. Acesso em: 12 jun. 2016. 
[16] CORRAR, J., L.; PAULO, E.; DIAS FILHO, M. J.. Análise Multivariada: Para os Cursos de Administração, Ciências Contábeis e Economia. São Paulo: Atlas, 2014. 586 p.

[17] OUYANG, Y. Evaluation of river water quality monitoring stations by principal component analysis. Water Research, v. 39, n. 12, p. 2621-2635, 2005. Disponível

em:

<http://linkinghub.elsevier.com/retrieve/pii/S0043135405001788>. Acesso em: 10 jul. 2016.

[18] MINGOTI, Sueli Aparecida. Análise de dados através de métodos de estatística multivariada: uma abordagem aplicada. 2. ed. Minas Gerais: Ufmg, 2013. $295 \mathrm{p}$

[19] FUNDAÇÃO ESTADUAL DE PROTEÇÃO AMBIENTAL HENRIQUE ROESSLER - FEPAM. Monitoramento da Qualidade da Água da Região Hidrográfica das Bacias Litorâneas. Site oficial da FEPAM. 2016. Disponível em: http://www.fepam.rs.gov.br/qualidade/monitor_agua_litoral.asp. Acesso em: 01 jun. 2016.

[20] COMITÊ DE GERENCIAMENTO DA BACIA HIDROGRÁFICA DO RIO CAMAQUÃ - CGBHRC. Site oficial da CGBHRC. 2016. Disponível em: http://www.comitecamaqua.com/index.php. Acesso em: 01 jun. 2016.

[21] COMPANHIA DE TECNOLOGIA DE SANEAMENTO AMBIENTAL. 1987. Guia de coleta e preservação de amostras de água. CETESB, São Paulo, SP, Brasil.

[22] AMERICAN PUBLIC HEALTH ASSOCIATION - APHA. Standard methods for the examination of water and wastewater. $21^{\text {a }}$ ed. Washington: American Public Health Association. APHA/AWWA/WEF, 2005.

[23] HAIR, F.J.; BLACK, W. C.; BABIN, B. J.; ANDERSON, R. E.; TATHAM, R. L.. Análise multivariada de dados. $6^{\text {a }}$ ed. Porto Alegre: Bookman, 2009.

[24] JOLLIFFE, I.T. Principal Component Analysis. 2. ed. New York: Springer, 2002. $518 \quad$ p. $\quad$ Disponível <http://cda.psych.uiuc.edu/statistical_learning_course/JolliffeI.Principal Component Analysis(2ed., Springer,2002)(518s)_MVsa_.pdf>. Acesso em: 13 mai. 2016

[25] BERTOSSI, A. P. A; CECÍlIO, R. A; NEVES, M. A.; GARCIA, G. O. Qualidade da água em microbacias hidrográficas com diferentes coberturas do solo no sul do Espírito Santo. Revista Árvore, [s.1.], v. 37, n. 1, p.107-117, 2013. Disponível em: <http://www.scielo.br/scielo.php?script=sci_arttext\&pid=S010067622013000100012>. Acesso em: 12 jun. 2016.

[26] VON SPERLING, M. Estudos e modelagem da qualidade da água de rios. Belo Horizonte: DESA/UFMG, 2007, 588 p. (Coleção Princípios do Tratamento Biológico de Águas Residuárias, v.7)

[27] ZIMMERMANN, Ciro Maurício; GUIMARÃES, Orliney Maciel; PERALTA-ZAMORA, Patricio Guillermo. Avaliação da qualidade do corpo hídrico do rio Tibagi na região de Ponta Grossa utilizando análise de componentes principais (PCA). Química Nova, [s.1.], v. 31, n. 7, p.1727-1732, 2008. FapUNIFESP (SciELO). http://dx.doi.org/10.1590/s0100$40422008000700025 . \quad$ Disponível em: $<$ http://www.scielo.br/scielo.php?script=sci_arttext\&pid=S010040422008000700025>. Acesso em: 25 jul. 2016.

[28] GUEDES, H. SILVA, D.D; ElESBON, A.A, A; RIBEIRO, C. B.M; MATOS, A.T; SOARES, J.H.P. Aplicação da análise estatística multivariada no estudo da qualidade da água do Rio Pomba, MG. Revista Brasileira de Engenharia Agrícola e Ambiental - Agriambi, Campina Grande, v. 16, n. 5, p. 558-563, mai. 2012. Disponível em: <http://www.scielo.br/scielo.php?script=sci_arttext\&pid=S1415 -

$43662012000500012 \& \operatorname{lng}=$ en\&nrm=iso>. Acesso em: 10 mai. 2016.

[29] SENGORUR, B; KOKLU, R; ATES, A. Water Quality Assessment Using Artificial Intelligence Techniques: SOM and ANN - A Case Study of Melen
River Turkey. Water Quality, Exposure and Health, [s.1.], v. 7, n. 4, p.469-490, 7 mar. 2015. Disponível em: <https://www.researchgate.net/profile/Rabia_Koklu/publications>. Acesso em: 20 mai. 2016.

[30] SMETI, E. M.; KORONAKIS, D. E.; GOLFINOPOULS, S. K. Control charts for the toxicity of finished water -Modeling the structure of toxicity, Water Research, v. 41, n. 12, pp. 2679-2689. jun. 2007. Disponível em: <http://www.ncbi.nlm.nih.gov/pubmed/17442368 >. Acesso em: 20 jun. 2016.

[31] FERREIRA, D. F. Estatística Multivariada. 2. Ed. Lavras: UFLA, 2011.

[32] FRANÇA, M. S. Análise estatística multivariada dos dados de monitoramento de qualidade de água da bacia do alto Iguaçu: uma ferramenta para a gestão de recursos hídricos. 2009. 166 f. Dissertação (Mestrado) - Curso de Programa de Pós-graduação em Engenharia de Recursos Hídricos e Ambiental, Universidade Federal do Paraná, Curitiba, 2009. Disponível em: $<$ http://acervodigital.ufpr.br/bitstream/handle/1884/23052/DissertacãoMarianne Schaefer Franca.pdf? sequence=1>. Acesso em: 13 mai. 2016. 